How to Cite

Giri, M. K. W. (2019). Immunological side in overtraining exercise. International Journal of Health \& Medical Sciences, 2(1), 1-

6. https://doi.org/10.31295/ijhms.v2n1.52

\title{
Immunological Side in Overtraining Exercise
}

\author{
Made Kurnia Widiastuti Giri \\ Universitas Pendidikan Ganesha, Singaraja, Indonesia \\ Email:drnia82@gmail.com
}

\begin{abstract}
The current paper is a kind of review study. It reviewed the immunological side in overtraining exercise. The method used in reviewing the content was a descriptive qualitative method. The review itself was based on theoretical views. As the results, it is stated that the beneficial effects of regular exercise on the prevention and therapy of lifestyle, related disease, has been established for a long time. However, it is often misinterpreted, so that people conduct the over-exercise activity. The overtraining, that is a phenomenon of conducting the exercise in excessive duration, frequencies, and intensity impacts the damage of myocardial. Furthermore, the complexity and pleiotropic effects of exercise remain an area of intense research. Therefore, in order to complete the frameworks of overtraining theory, future research, that is to confirm and to investigate pathomechanism of organs damage, is needed to be conducted.

Keywords---biomolecular, damage, myocardial, overtraining, oxidative.
\end{abstract}

\section{Introduction}

In the medical sciences, the importance of review articles is rising (Biddle \& Asare, 2011). When clinicians want to update their knowledge and generate guidelines about a topic, they frequently use reviews as a starting point. The value of a review is associated with what has been done, what has been found and how these findings are presented. Related to this review article, we try to reveal the immunological side in overtraining (Oxman et al., 1994). Previous studies have established a concept that biomarkers have been interested in the studies of physical exercise. In order to measure performance, it is undeniable, biomarkers are needed. Furthermore, they are used to identify the progress of training as well as for identifying overtraining. Nowadays, the biomolecular aspect of exercise has been concerned in several studies related to the benefits of proportional exercise and the possibility of overtraining exercise that has got many disadvantages for many organs in human's body (Wallis et al., 2010).

It is known that regular physical exercise has got many health benefits including a lowered threat of all-cause mortality along with a reduced risk of cardiovascular disease, cancer, and diabetes (Chang et al., 2013). Paradoxically, it is also clear that contracting skeletal muscles generate free radicals and that prolonged and intense exercise can result in oxidative damage to cellular constituents (Fatouros et al., 2006). During the last few decades, our knowledge about the biological implications of exercise-induced oxidative stress has expanded rapidly. It has become the biomolecular effects in our cell. Indeed, it is now appreciated that while high levels of free radicals can damage cellular components, but low to moderate levels of oxidants play multiple regulatory roles in cells, such as the control of gene expression, regulation of cell signaling pathways, and modulation of skeletal muscle force production.

Because of recent advances in the field of exercise and oxidative stress, this paper is viewed as an appropriate time, that is to summarize some of the major principles of exercise-induced oxidative stress and its impact on skeletal muscle function. Our approach provides a critical synopsis of myocardial effects of exercise, especially when it has overtraining aspects. We will begin with an overview of a biomolecular aspect of exercise, the concept of oxidative stress, overtraining also myocardial damage. Finally, I will suggest some directions for future research in this field.

ISSN 2632-9433

Received Jan 20, 2019 / Accepted Jun 18, 2019 / Published Jul 05, 2019 


\section{Methodology}

Use of proper methodologies in review articles is important in that readers assume an objective attitude towards updated information. Therefore, in this section, I describe the method I applied. As have been inferred above, this paper is categorized as a review paper. As a review paper, the critical literature review method was applied. It sought to critically review the literature on immunological sciences and overtraining to suggest avenues for further researches that relate to myocardial effects of exercise. Data of the current research were collected with impartial cross-referencing of papers published in all major journals in the health sciences area. In presenting the review, I used the descriptive qualitative method.

\section{Results and Discussion}

\section{Biomolecular Aspects of Exercise}

A biomarker (biological marker) is a measurable product or substance used as an indicator of the biological state. Its aim is to objectively determine the body's physiological or pathological processes. In exercise, biomarkers are key parameters to assess the impact of exercise on different systems, tissues, and organs (Tremblay et al., 1994). Furthermore, biomarkers used to measure the impact of training on the long term as a chronic effect or the acute effect of exercise. The value or concentration of a biomarker depends on many factors, such as the training status of the subject, the degree of fatigue, the type, and duration of exercise, apart from age, and gender, etc.

In the other side, biomarkers could be a sign of biological function that acts as a physiological process and also initiation pathological process (Strimbu \& Tavel, 2010). Cytokine is one biomarker that often used in several sports medicine research. Kind of interleukin categorized pro-inflammatory and anti-inflammatory. Especially in exercise, there is Interleukin 6 (IL 6). IL 6 is a kind of token called pleiotropic that acts as an anti-in-inflammatory that produced by a muscle called as a myokine. The following figure describes how IL 6 is operating in the human body. The figure explains IL6 is released by the skeletal muscles. It affects a variety of biochemical processes in the sport. Various communications of cytokines occur with IL6 which secreted by skeletal muscles. Fat oxidation, respiratory chain, and angiogenesis in exercise is influenced by secretion of IL6 (Skulachev, 1991).

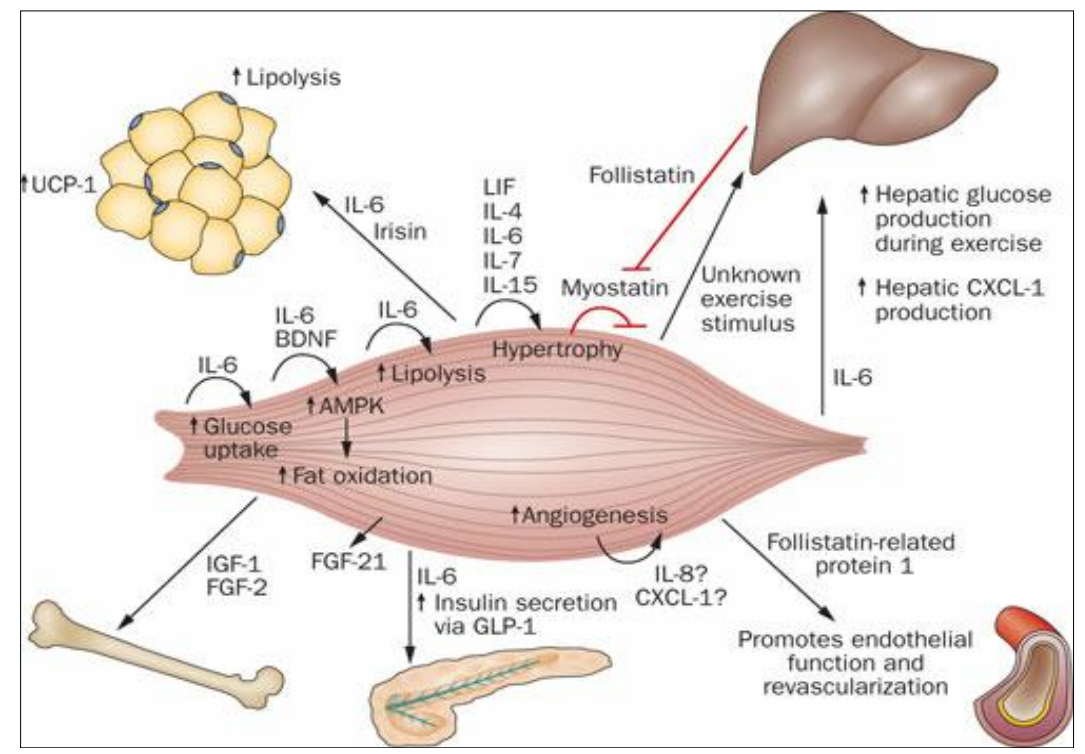

Figure 1. Skeletal muscle as a secretory organ

Moreover, IL6 is a pleiotropic cytokine which has an important role in the regulation of the immune system and metabolic exercises. During the exercise, IL6 is produced and released from skeletal muscle contraction. The amount of IL-6 is released related to the duration and intensity of exercise. IL6 itself is a cytokine which first appeared in circulation after starting the exercise and has increased the most prominent compared to other cytokines in response 
to exercise. Several studies have demonstrated the role of IL6 as sensor muscle energy and suggest a potential role as a biomarker of overtraining (Di Raimondo et al., 2016).

IL6 in the exercise plays an important role in the activation of $A M P K . A M P K$ is a protein that is important in the respiratory chain. IL6 is also instrumental to induce anti-inflammatory cytokines such as IL-10 and IL1ra as well as to suppress the expression of proinflammatory cytokine TNF $\alpha$ (Hunter \& Jones, 2015).

\section{Oxidative Stress}

The human body, through cells inside the body, produces free radicals and Reactive Oxygen Species (ROS) as part of the metabolic processes. Related to that, exercise can increase the amount of ROS through a variety of mechanisms in cell metabolism pathways. ROS are divided into two types, namely oxidants and free radicals. Although the process is different from one another, the activity of those two compounds has the same result effects (Zweier \& Talukder 2006).

Furthermore, oxidants, in terms of chemistry, is the electron acceptor compounds (electron acceptors). They are compounds that can attract electrons $(\mathrm{Fe}+++)$. Free radicals as an atom or molecule have got one or more unpaired electrons. The free radicals are produced in the process of aerobic cellular metabolism and have a role as a key mediator in the regulation of signaling processes. Oxidative stress reflects an imbalance between the production of ROS and antioxidants are adequate (Gomes, 2014).

The facts as mentioned above, or, the adverse conditions, can cause damage to cells and tissues that are involved in pathophysiological processes, including aging, exercise, inflammation, heart disease, neurodegenerative diseases, and malignancies. Study related to the topic has increased in some health scientific studies within the past 30 years. The study focuses on medical research about ROS in physical exercise that is to increase ROS as the effects of acute exercise. Increasing an excessive amount of ROS has been proved as a result of oxidative stress in excessive exercise (vigorous and also overload). Skeletal muscle oxidative stress in the exercise is described through various mechanisms. One of the mechanisms itself is the ROS as signaling molecules. Another mechanism that increases ROS is through the influence of gene that is through a redox-sensitive transcription pathway. The Figure 2, as presented following, is the regular exercise which raises hormesis and reduces oxidative stress, as well as to protect the body from disease attack, and to improve the performance and the quality of life. Vigorous exercise and overtraining, as have been described, increase oxidative stress and risk for disease.

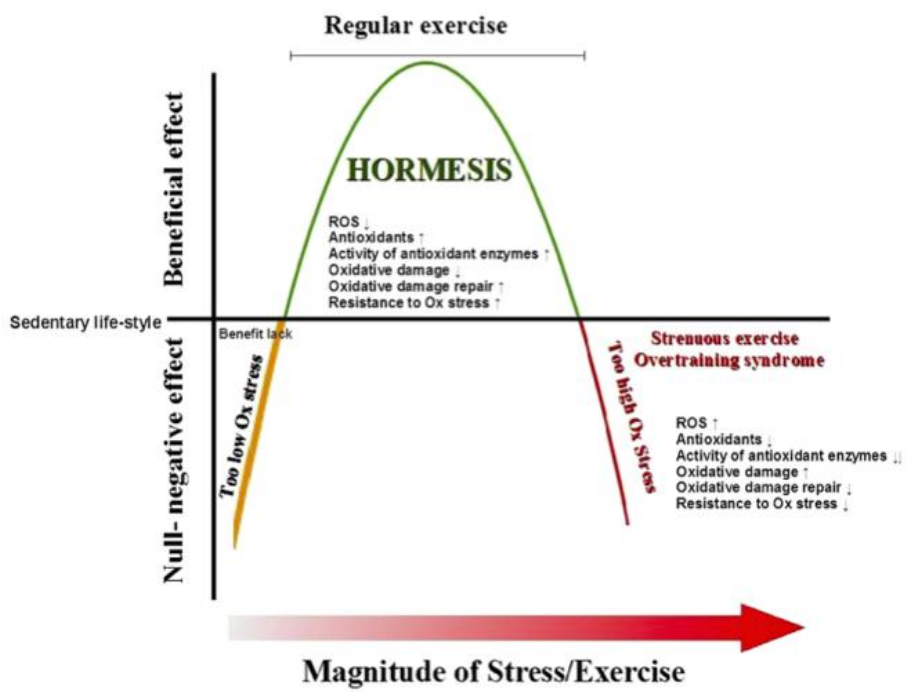

Figure 2. Hormesis concept in exercise

In particular, the relationship between exercise and oxidative stress are very complex, depending on the type of exercise program, intensity, and duration of the exercise (Radak et al., 2005). Training with moderate intensity has been shown to be beneficial for health. In contrast, high-intensity exercises cause an increase in oxidative stress. Physiologically, exercise is a stimulus that is required to increase the regulation of endogenous antioxidants (hormesis).

Moreover, increased intensity of exercise will lead to an increase in the stress of oxidative and free radicals. ROS in the exercise, as has been investigated, occurred as a result of several different mechanisms. Those mechanisms are 
(1) the existence of ROS released from the metabolic processes enzymes in mitochondria; (2) the occurrence of hypoxia and reoxygenation process various capillary endothelium during intensive exercise. It also occurs in the pathogenesis of the cardiovascular disease, and (3) the occurrence of oxidative burst of inflammatory cells that occurs as a result of muscle damage in training with high intensity and with a load, or even overload. Damage to the muscle cells causing the fragmentation of the muscle cells, so it will stimulate macrophages through Toll-Like Receptor 4 (TLR-4). The stimulation will trigger macrophages to release proinflammatory cytokines. Along with the increased of oxidative phosphorylation in exercise, then it is known that there is also the formation of ROS increased. ROS itself, cannot be denied, produced from 2 up to $5 \%$ oxygen consumption by mitochondria.

The release of catecholamines in exercise also triggers the production of ROS. Some other sources in the formation of ROS is a prostanoid metabolism, xanthine oxidase, NADPH oxidase, and several other secondary sources such as free radicals that are released by mitochondria. The process is a result of the reconstruction of cell damage. The ROS will try to take electrons from other molecules. As a result, capturing electrons in the molecule will cause a disturbance in the function of molecules. It can even damage the structure of the molecule biochemistry.

ROS can be neutralized by the influence of anti-ROS. Anti-ROS is useful as an electron donor. In normal circumstances, in accordance with the law of homeostasis, ROS and anti-ROS are expressed in equilibrium. ROS, with the ability to take electrons from the cell components, will cause malfunction and even damage to the various components of the cells. Such damage may include cell membranes, particularly containing phospholipids, DNA, protein molecules. It also impacts the damage of enzymes, receptors, antibodies, matrix, and the cytoskeleton. Furthermore, ROS produces mitochondrial of PMN, monocytes, or macrophages.

The presence of ROS in exercise can be a benefit or be dangerous. It depends on the concentration of ROS, ROS exposure duration, and status of individual exercise (type, intensity, duration, and frequency of exercise) (Powers et al., 2011). Related to the thoughts described above, overtraining is stated as the factor that causes an increase of ROS in large quantities. As the impact, it cannot be covered through the buffer system of endogenous antioxidants. This causes oxidative stress that leading to muscle weakness and fatigue, mutation of DNA, lipid peroxidation, mitochondrial dysfunction, and apoptosis or necrosis. ROS is generated during a routine exercise with a proportional exercise program, that will increase the level of adaptation by increasing antioxidant capacity, mitochondrial biogenesis, insulin sensitivity, protection of cells and aerobic capacity of skeletal muscle.

\section{Overtraining Exercise}

Endurance training can increase oxygen consumption. It can form free radicals and may result in damage to the muscle or other body tissues. The human body itself has got antioxidant that defenses and fights the establishment of free radicals. Training or exercise are the main factors that can prevent the aging process. However, it must be done with the proper dose in order to prevent a condition called overtraining which can be harmful to health. Exercise is an important factor in health and could delay the aging process. Many studies show evidence that regular physical activity or exercise decreases the risk of people experiencing a disease such as coronary heart disease, type 2 diabetes, and hypertension. It has been also proven that exercise can reduce morbidity and mortality, not only to improve survival but also increase the productivity of life. Blood pressure in hypertension condition can only be decreased by doing regular physical activity. Furthermore, such activity will decrease fat and cholesterol levels, increasing the levels of High-Density Lipoprotein (HDL), and increases sensitivity to insulin. The other benefits of the physical activity are increased energy levels, improving self-confidence, improving strength and ability to perform daily activities, increasing muscle mass, and decreasing body fat. The positive effects make the exercise as the best anti-aging therapies. Therefore it is stated that exercise and physical activity are very useful to improve the health and quality of life. For the purpose of staying healthy, World Health Organization (WHO) and also American Heart Association (AHA) recommend that adult should take at least 30 minutes of physical activity or exercise as much as 3 to 5 times per week.

Unfortunately, in many cases, people conducted an exercise with overdose, and with a wrong way that may be harmful to health. The exercise carried out by excessive, somehow, is because of the misinterpretation. Some people think that exercise is healthy for health so that they have a perception that better to do more exercise and exhaustive. In fact, excessive exercise, which is also called as overtraining, will endanger health.

Overtraining can also occur when there is an imbalance between (1) training and recovery, (2) training and capacity training skills, (3) stress and stress tolerance. Short-term overtraining will occur over several days to two weeks with symptoms of fatigue, decreased maximum training capacity, and the inability to compete. Recovery occurs a few days, with a better prognosis. Long-term overtraining occurs over several weeks to months which resulted in overtraining syndrome. Stacking excessive training with other fatigue, causing a decrease in the 
maximum training capacity, feeling disorders, stiffness and pain of muscles, and decreased the ability to compete long term. Complete recovery may take weeks to months.

There is also a change in the components of the blood, hormone levels, and spending on nocturnal urine catecholamine. Furthermore, overtraining occurs when the intensity and volume of training exceed the capacity of the recovery, which then causes changes in emotional, behavioral and physical condition. Because of overtraining, there will also be a decrease in fitness and physical strength. Increased strength and fitness occur only during the period of rest after strenuous training. It can take 12-24 hours. If the rest period is not enough then regeneration will not occur. If the imbalance between weight training and proper rest is not sufficient to continue the performance will persist or even declined. Mild overtraining requires only a few day-rest, or by doing less activity until recovery occurs. If one still do the training, there will be an accumulation of fatigue that would settle beaming-weeks and even months.

Overtraining is preferred to occur in people who experience physical and psychological stress, such as during menstruation and malnutrition. It is a common problem in bodybuilding athletes and dieters who also conduct training with high intensity and reducing food intake. Some cases of severe overtraining occur more complex by

doing excessive training, as well as a too short-recovery time. It involves many factors including the burden of training and lack of recovery time (Brooks, 2013). Several possible mechanisms of overtraining are (1) the occurrence of minor trauma is faster than the healing process, (2) the use of amino acids are bigger than the intake, or often called protein deficiency, (3) the body becomes calorie deficiency, (4) levels of the hormone cortisol increase in a long time, (5) the body length is in a catabolic, more than in an anabolic, (6) stretching on the nervous system that happens continuously during the training, and (7) the buildup of free radicals (Merry \& Ristow, 2016).

Furthermore, overtraining is stated as a complex syndrome as well as a mixture of signs and symptoms of mental fatigue feeling that looks at physical fatigue and decreased performance. It increases basal metabolic rate, weight loss with a negative balance of nitrogen. Overtraining also triggers the breakdown of muscle cells that expressed by an increase in cytokines that lead to muscle growth disorder which is determined by certain genes.

\section{Myocardial Damage}

In proportional exercise, there is an amount of a physiological process of organs, such as muscle, kidney, and heart. Myocardium has similar histologically forms with skeletal muscle. Myocardium itself has a complex function related to the cardiorespiratory functions. Related to cardiorespiratory functions, physical activity is needed for gaining Oxygen $\left(\mathrm{O}_{2}\right)$. It is supported by cardiorespiratory function.

The average of $\mathrm{O} 2$ consumption by the human body, for young and adult, is about $3.5 \mathrm{ml} / \mathrm{kg} / \mathrm{min}$. $20 \%-25 \%$ of that is gained through resting the skeletal muscle. In lean, untrained healthy adults, VO2max is typically $10-15$ times resting values. In elite endurance-trained athletes, VO2 max values can exceed $85 \mathrm{ml} / \mathrm{kg} / \mathrm{min}$ (Mcardle et al., 2010; Hawley et al., 2014). Counting such thoughts, it is concluded that VO2 max is determined by combining capacities of the central nervous system to recruit motoric units. Associated with large increases in $\mathrm{O} 2$ consumption during maximal exercise, humans are the peak for cardiac output (Q) and ventilation of 40 and $200 \mathrm{l} / \mathrm{min}$ (Hawley et al., 2014).

Some other physiological changes in the heart, in addition, to increase cardiac output, is by (1) increasing oxygen consumption, stroke volume, systolic blood pressure, and (2) decreasing peripheral vascular resistance and heart rate. In contrast to aerobic exercise, anaerobic exercise will increase oxygen consumption and cardiac output. On the other hand, there is an increase in blood pressure, peripheral resistance and heart rate. From these two different mechanisms on the exercises, it is stated that the aerobic exercise occurred in the left ventricular that is when left ventricular is pressured overload.

\section{Conclusion}

The beneficial effects of regular exercise on the prevention and therapy of lifestyle-related disease are long established. Nowadays, the biomolecular based for adaptive changes in skeletal muscle and metabolic function has been shown in several studies. The complexity and pleiotropic effects of exercise remain an area of intense research. According to the harm of overtraining, when exercise is conducted in excessive duration, frequencies, and intensity, it needs to be researched, or to investigate whether the pathomechanism of organs is damaged or not.

Myocardium as an important tissue constructs one of the organs functionally in cardiorespiratory performance of athletes (Leeuwenburgh et al., 1997). An overtrained-athlete could get decreased in their performance because of the 
damages of some cells in the body. Pathomechanism of overtraining is still debated. Therefore, the study of biomolecular is still needed to be conducted in order to complete the frameworks of overtraining theory.

Myocardial damage in overtraining is reviewed based on theoretical frameworks included cytokines expressions of macrophage elucidated, pathomechanism of inflammation in endosteal, muscles, and myocardium. The exceeded production of ROS in exercise by mitochondria and also movement itself impacts some disturbance of the equal conditions of radicals and endogenous antioxidants. It is also assumed that damage to skeletal muscles and myocardium are related to the response of macrophage and mitochondria. Finally, it is stated that studies of overtraining would deliver an improvement of awareness to avoid overtraining itself and to create a proportional program of training in exercise. Therefore, it is suggested that further research related to the current topic can be conducted for the improvement of health sciences theory.

Acknowledgments

This work was supported by the Research Fund provided by Universitas Pendidikan Ganesha, Bali, Indonesia.

\section{References}

Biddle, S. J., \& Asare, M. (2011). Physical activity and mental health in children and adolescents: a review of reviews. British journal of sports medicine, 45(11), 886-895. 10.1001/jama.1994.03520170077040

Chang, Q., Miao, X., Ju, X., Zhu, L., Huang, C., Huang, T. \& Gao, C. (2013). Effects of pulse current on endurance exercise and its anti-fatigue properties in the hepatic tissue of trained rats. PloS one, 8(10), e75093. https://doi.org/10.1371/journal.pone.0075093

Di Raimondo, D., Tuttolomondo, A., Musiari, G., Schimmenti, C., Angelo, A., \& Pinto, A. (2016). Are the myokines the mediators of physical activity-induced health benefits?. Current pharmaceutical design, 22(24), 3622-3647.

Fatouros, I. G., Kambas, A., Katrabasas, I., Leontsini, D., Chatzinikolaou, A., Jamurtas, A. Z., ... \& Taxildaris, K. (2006). Resistance training and detraining effects on flexibility performance in the elderly are intensity-dependent. The Journal of Strength \& Conditioning Research, 20(3), 634-642.

Gomez-Cabrera, M. C. (2014). Antioxidants in skeletal muscle physiology, a radically different approach. Free Radical Biology and Medicine, 75, S1-S2. https://doi.org/10.1016/j.freeradbiomed.2014.10.596

Hawley, J. A., Hargreaves, M., Joyner, M. J., \& Zierath, J. R. (2014). Integrative biology of exercise. Cell, 159(4), 738749. https://doi.org/10.1016/j.cell.2014.10.029

Hunter, C. A., \& Jones, S. A. (2015). IL-6 as a keystone cytokine in health and disease. Nature Immunology, $16(5), 448$. https://doi.org/10.1038/ni.3153

Leeuwenburgh, C., Hollander, J., Leichtweis, S., Griffiths, M., Gore, M., \& Ji, L. L. (1997). Adaptations of glutathione antioxidant system to endurance training are tissue and muscle fiber specific. American Journal of Physiology$\begin{array}{llll}\text { Regulatory, Integrative } \quad \text { and Physiology, 272(1), } & \text { R363-R369. }\end{array}$ https://doi.org/10.1152/ajpregu.1997.272.1.R363

McArdle, W. D., Katch, F. I., \& Katch, V. L. (2010). Exercise physiology: nutrition, energy, and human performance. Lippincott Williams \& Wilkins.

Merry, T. L., \& Ristow, M. (2016). Mitohormesis in exercise training. Free Radical Biology and Medicine, 98, 123-130. https://doi.org/10.1016/j.freeradbiomed.2015.11.032

Oxman, A. D., Cook, D. J., Guyatt, G. H., Bass, E., Brill-Edwards, P., Browman, G., \& Haynes, B. (1994). Users' guides to the medical literature: VI. How to use an overview. Jama, 272(17), 1367-1371. http://dx.doi.org/10.1136/bjsports2011-090185

Powers, S. K., Ji, L. L., Kavazis, A. N., \& Jackson, M. J. (2011). Reactive oxygen species: impact on skeletal muscle. Comprehensive Physiology, 1(2), 941-969. http://doi.org/10.1002/cphy.c100054

Radak, Z., Chung, H. Y., \& Goto, S. (2005). Exercise and Hormesis: Oxidative stress-related adaptation for successful aging. Biogerontology, 6(1), 71-75.

Skulachev, V. P. (1991). Fatty acid circuit as a physiological mechanism of uncoupling of oxidative phosphorylation. FEBS letters, 294(3), 158-162. https://doi.org/10.1016/0014-5793(91)80658-P

Strimbu, K., \& Tavel, J. A. (2010). What are biomarkers? Current Opinion in HIV and AIDS, 5(6), 463.

Tremblay, A., Simoneau, J. A., \& Bouchard, C. (1994). Impact of exercise intensity on body fatness and skeletal muscle metabolism. Metabolism, 43(7), 814-818.

Wallis, R. S., Pai, M., Menzies, D., Doherty, T. M., Walzl, G., Perkins, M. D., \& Zumla, A. (2010). Biomarkers and diagnostics for tuberculosis: progress, needs, and translation into practice. The Lancet, 375(9729), 1920-1937. https://doi.org/10.1016/S0140-6736(10)60359-5

Zweier, J. L., \& Talukder, M. H. (2006). The role of oxidants and free radicals in reperfusion injury. Cardiovascular Research, 70(2), 181-190. https://doi.org/10.1016/j.cardiores.2006.02.025 